\title{
Characterization of spinal cord injury among patients attended in a rehabilitation center: experience of the department of physical medicine and rehabilitation of the military instruction hospital mohamed V-rabat/morocco
}

\begin{abstract}
Introduction: Spinal cord injury is a frequent cause of hospitalization in a Physical Medicine and Rehabilitation Department. Many complications can be observed in these patients. In Morocco, there is no epidemiological register for patients with spinal cord injury and for the complications they encounter. Our study aims to determine the socio-demographic and clinical characteristics especially the complications observed in spinal cord injury population and their treatments.
\end{abstract}

Materials and methods: We conducted a retrospective study in which all patients hospitalized for spinal cord injury in the physical medicine and rehabilitation department of the Mohammed V Military Hospital in Rabat have been listed. The socio-demographic characteristics, the severity of the deficit according to the American Spinal Injury Association (ASIA) scale, the aetiology, the complications observed during the hospitalization and during the follow-up, and the treatments used have been noted.

Results: 116 in patients with spinal cord injury have been treated in the Physical Medicine and Rehabilitation Department of the Mohammed V Military Hospital in Rabat from 2008 to 2013 . The average age was 40.5 years and the sex ratio M/F was $4 / 1.53 \%$ were paraplegic and $47 \%$ were quadriplegic; $43,1 \%$ were ASIA A, $22,41 \%$ ASIA B, $18,96 \%$ ASIA C and $15,51 \%$ ASIA D). The aetiology was traumatic in $2 / 3$ of the cases. Complications were pressure ulcers in $42 \%$ of cases, urinary infections in $19 \%$, spasticity in $12 \%$, paraosteoarthropathy in $8 \%$, respiratory complications in $7 \%$, stiffness in $5 \%$, neuropathic pain in $4 \%$ and autonomous hyperreflexia in $3 \%$. After an average stay of 7.73 weeks, $47 \%$ of patients returned to partial activities and hobbies, $31 \%$ of defect engine recovery, $15 \%$ had a stable condition and $5 \%$ were lost to follow-up.

Discussion: Spinal cord injury is relatively common in the department of physical medicine and rehabilitation of the military hospital of Rabat. It affects a young population with male predominance. The aetiology is often traumatic and the complications are multiple.

Keywords: spinal cord injury, complication, management, rehabilitation, morocco
Volume 2 Issue 5 - 2017

\author{
Saloua Khalfaoui, Imad Bendeddouche EL, El \\ Abbassi \\ Department of Physical Medicine and Rehabilitation, Military \\ Instruction Hospital MohammedV, Morocco
}

\section{Correspondence: Saloua Khalfaoui, MohamedV University, Faculty of Medicine and Pharmacy of Rabat, Morocco, Tel +212612608298, Email Salaouine@hotmail.com}

Received: March 01, 2017 | Published: December 2I, 2017

\section{Introduction}

The constant evolution of knowledge, especially in the pathophysiology of cellular lesions, regularly generates new therapeutic approaches, some of them still controversial. The speed and quality of care provided to an injured person is a factor in reducing morbidity secondary to spinal cord injuries. Their application must be regularly evaluated within each hospital structure. In Morocco, there is no epidemiological register of spinal cord injuries and their complications. The aim of our study was to determine the sociodemographic characteristics of a population with spinal cord injury and also to list the clinical characteristics especially the complications it encounters and their treatments.

\section{Patients and methods}

We conducted a retrospective study. All patients hospitalized for spinal cord injury in the physical medicine and rehabilitation department of the Mohammed V Military Hospital in Rabat from January 2008 to February 2013 have been listed. These patients were transferred to the Department of Physical Medicine and Rehabilitation for rehabilitation, within one to two weeks of admission in neurosurgical or neurological departments. The sociodemographic characteristics, the severity of the deficit according to the American Spinal Injury Association (ASIA) scale, the aetiology, the complications observed during the hospitalization and during the follow-up and their treatments have been noted. 


\section{Results}

116 in patients with spinal cord injury have been treated in the Physical Medicine and Rehabilitation Department of the Mohammed V Military Hospital in Rabat from January 2008 to February 2013. The records of these patients have been studied. 87 of the patients were male (sex ratio $\mathrm{M} / \mathrm{F}$ is $4 / 1$ ) Figure 1 . Age groups [20-29] and [40-49] were the most represented with respective percentages of 19.60 and $25.49 \%$ with an average of $40.5 \pm 2$ years and extremes ranging from 12 to 70 years. In this series, $80 \%$ were active soldiers, $7 \%$ retired and $13 \%$ civilian. $53 \%$ had dorsal spinal cord injury and $47 \%$ had cervical spinal cord injury Figure 2 .

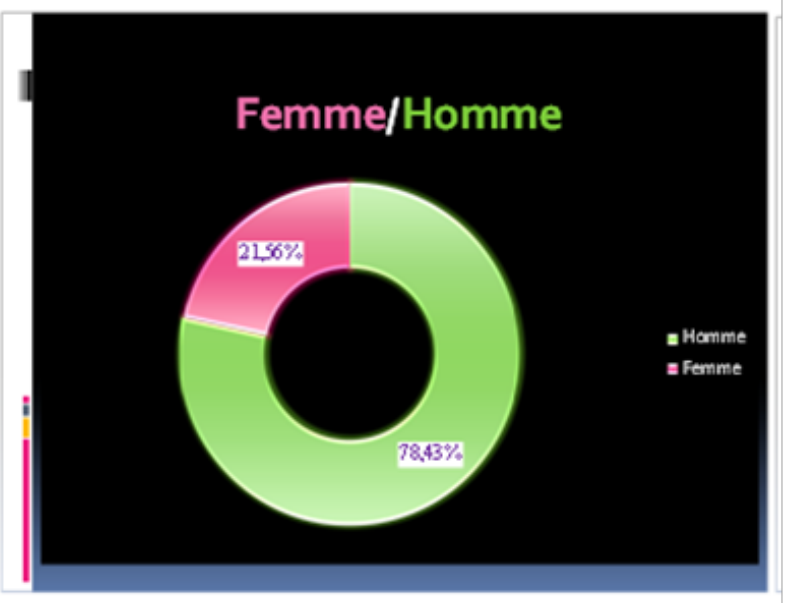

Figure I Spinal cord injury distributions by gender according to age groups.

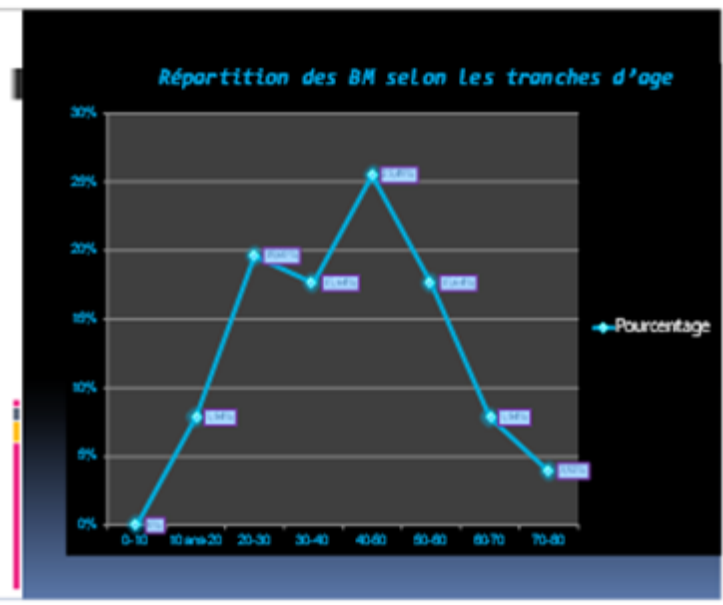

Figure 2 distributions of spinal cord injuries.

According to the American Spinal Injury Association (ASIA) scale, $43.1 \%$ of patients were ASIA A, $22.41 \%$ were ASIA B, $18.96 \%$ were ASIA C and $15.51 \%$ were ASIA D Figure 3 . The traumatic origin, including road traffic accidents, stabbing or bullet assault was found in 78 cases. 38 cases were of other origin such as tumours infections, inflammatory and degenerative origin Figure 4. Several complications were noted Figure 5.

i. Pressure ulcers in $42 \%$ of cases $(21 \%$ in the heel, $19 \%$ trochanteric, $14 \%$ occipital and $9 \%$ malleolar) with a median time to onset of 6 months Figure 6. ii. Urinary tract infections in $19 \% .42 \%$ were due to Escherichia coli. All these infections interested the low urinary tract with one case of orchiepididymitis. These infections were not treated in $1 / 5$ cases (Figure 7) (Figure 8).

iii. Spasticity in $12 \%$. This spasticity was treated by oral baclofen in $80 \%$ of cases and by intrathecal baclofen in only one case Figure 9.

iv. Paraosteoarthropathy was found in $8 \%$ of patients, it was located at the hip in $72 \%$ and at the knee in $28 \%$ (Figure 10) (Figure11).

v. Respiratory complications, stiffness, neuropathic pain and autonomic hyperreflexia were seen respectively in $7 \%, 5 \%, 4 \%$ and $3 \%$ of cases.

vi. After an average stay of $7.73 \pm 1$ weeks and a follow-up of 2 years, $47 \%$ partially returned to activities and recreation, $31 \%$ recovered from deficit, only $18 \%$ of patients returned to work, $15 \%$ kept a stable condition and $5 \%$ were lost to follow-up Figure 12

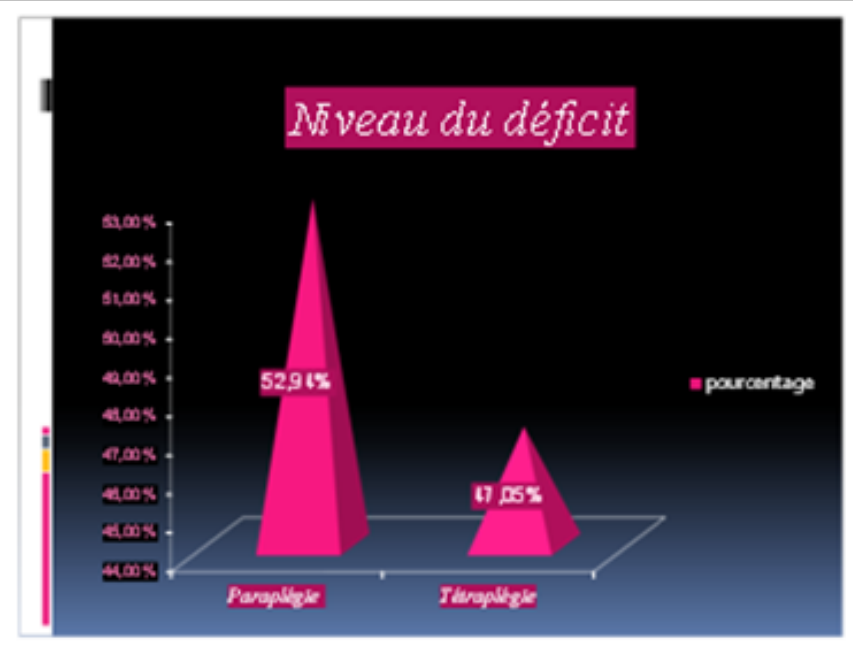

Figure 3 Deficit level.

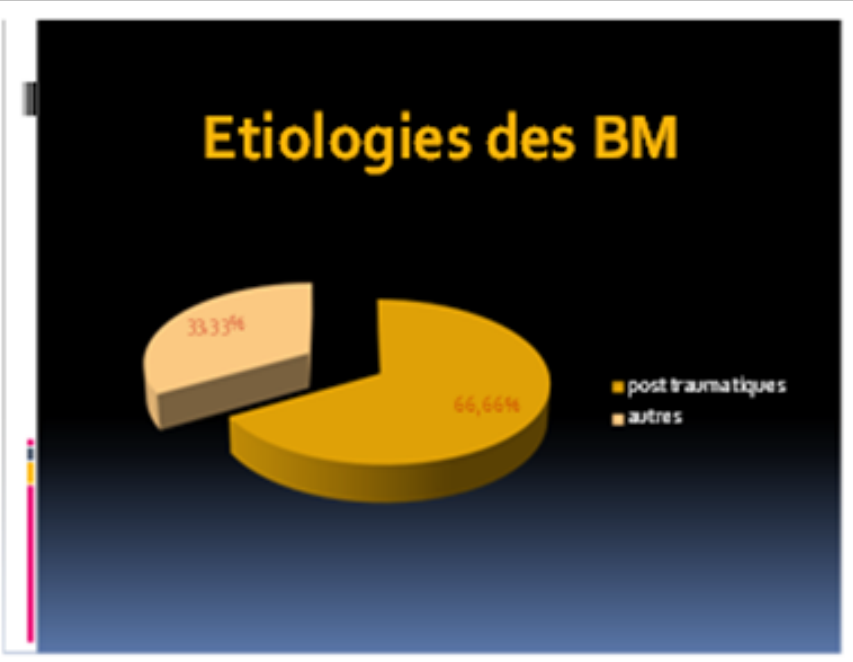

Figure 4 Etiology of spinal cord injured.

Citation: Khalfaoui S, Bendeddouche IEL, Abbassi E. Characterization of spinal cord injury among patients attended in a rehabilitation center: experience of the department of physical medicine and rehabilitation of the military instruction hospital mohamed V-rabat/morocco. Int Phys Med Rehab J. 20I7;2(5):269-273. DOI: 10.15406/ipmrj.2017.02.00060 


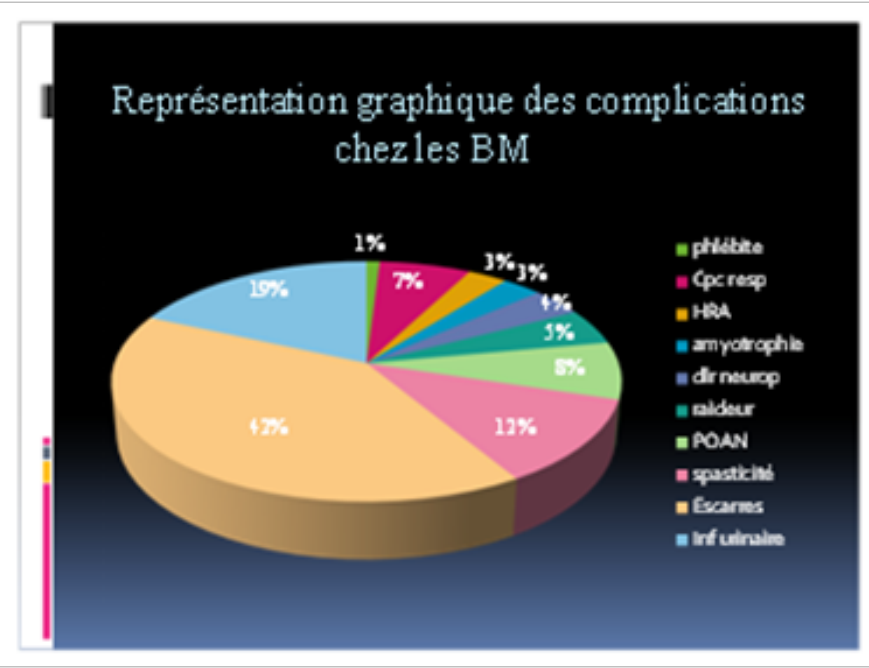

Figure 5 Complications in $\mathrm{SCl}$ patients.

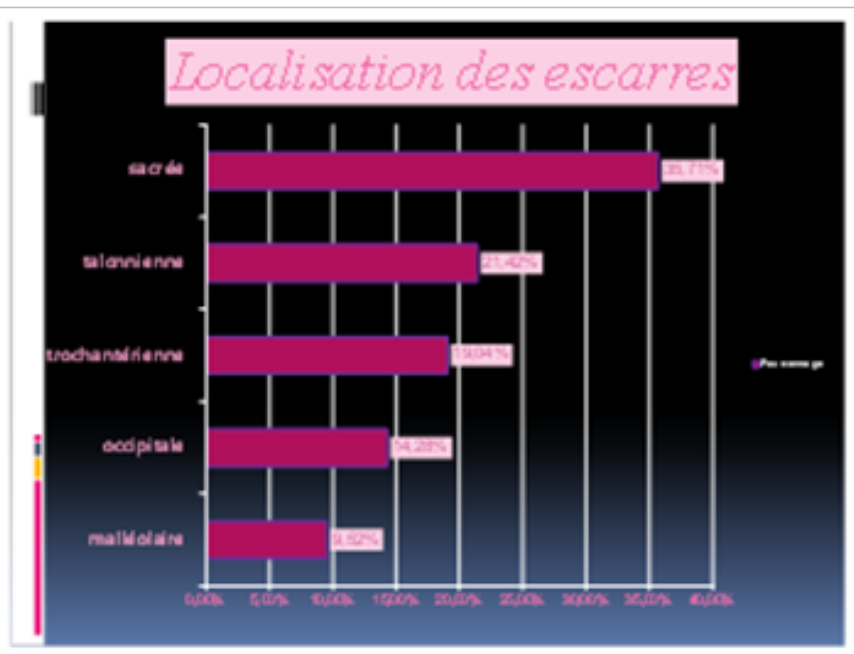

Figure 6 location of pressure ulcers.

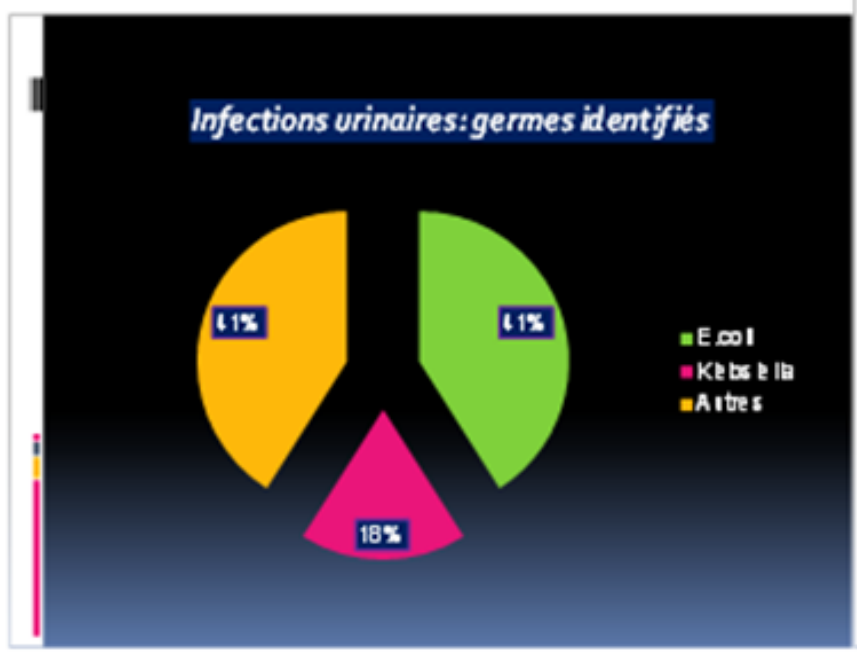

Figure 7 Urinary tract infections.

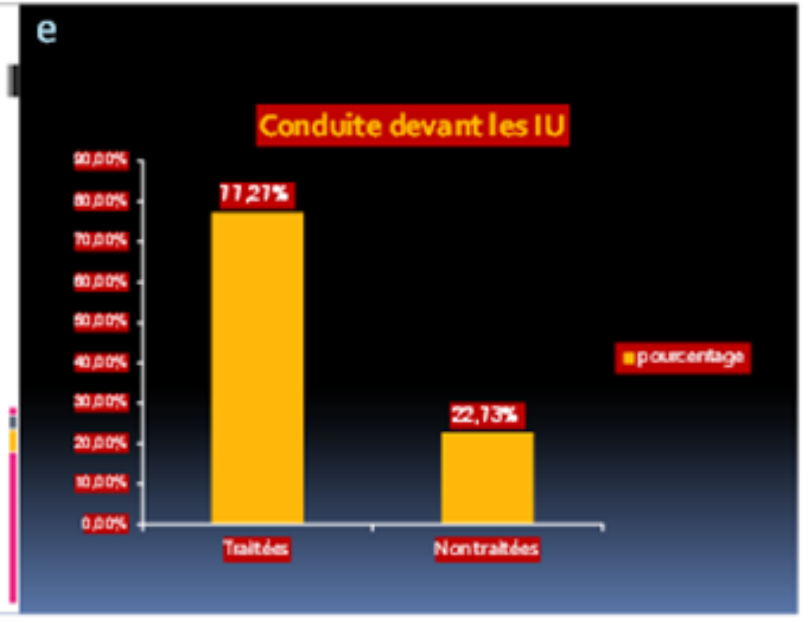

Figure 8 Urinary tract infections and treatment.

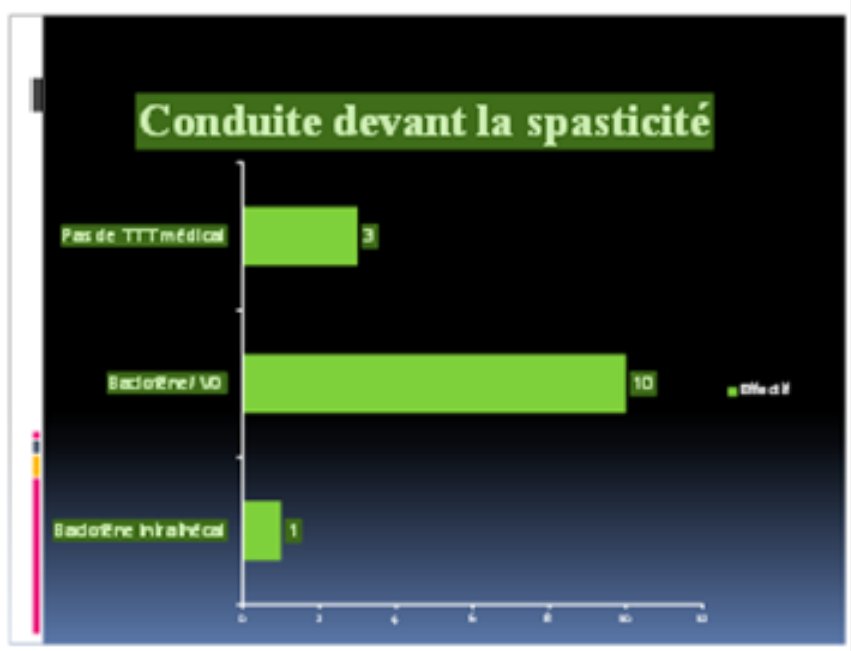

Figure 9 Spasticity and treatment.

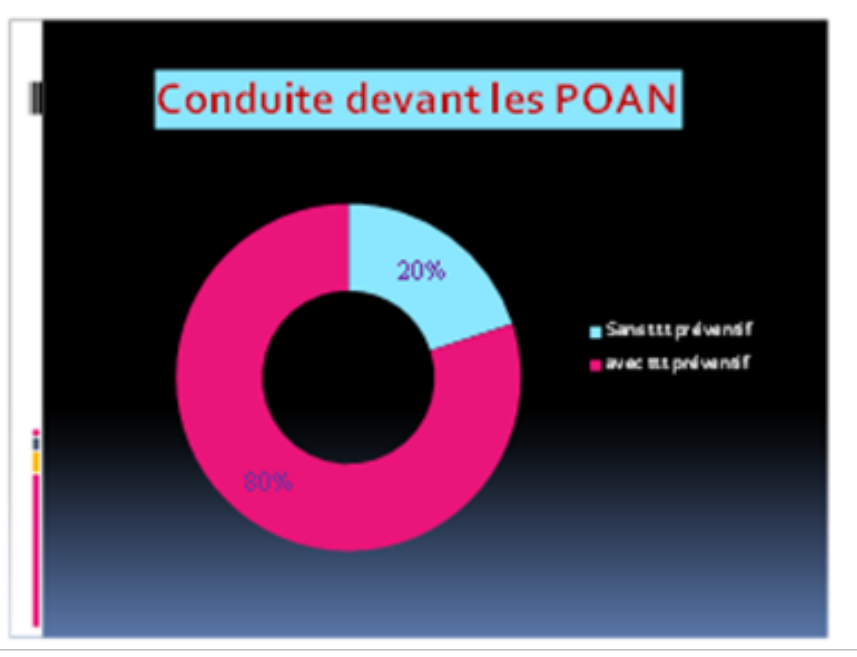

Figure 10 Para osteoarthropathy.

Citation: Khalfaoui S, Bendeddouche IEL, Abbassi E. Characterization of spinal cord injury among patients attended in a rehabilitation center: experience of the department of physical medicine and rehabilitation of the military instruction hospital mohamed V-rabat/morocco. Int Phys Med Rehab J. 20 I 7;2(5):269-273. DOI: 10.15406/ipmrj.2017.02.00060 


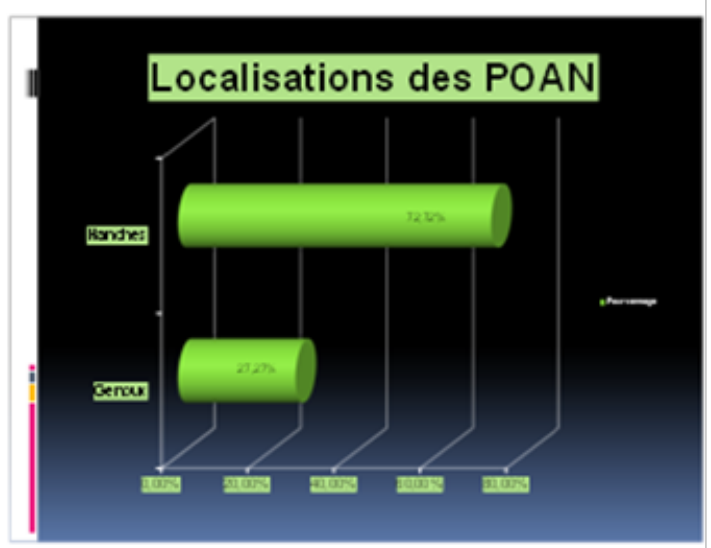

Figure I I Location of paraosteoarthropathy.

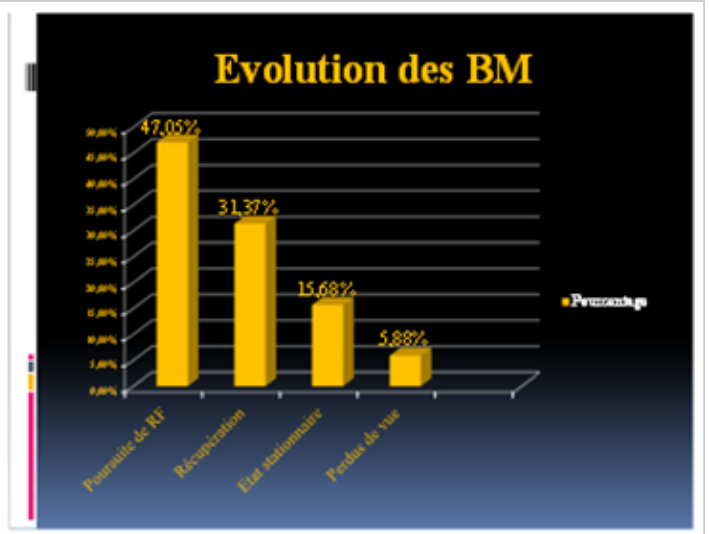

Figure $12 \mathrm{SCl}$ patients outcome.

\section{Discussion}

The mean age of onset of spinal cord injury is 30.7 years in France and 40.2 years in the United States with a sex ratio of $4 / 1 .{ }^{1}$ In our study, the age of onset is similar to USA and higher than France while the sex ratio is similar. The aetiologies are in more than $50 \%$ traumatic origin dominated by road accidents causing paraplegia and quadriplegia with almost identical percentages. ${ }^{2}$ Only $21.6 \%$ are returning to work. ${ }^{1}$ Our results showed higher percentage as $2 / 3$ of cases were from traumatic origin. The issue of the prevention of pressure sores is of major importance for the spinal cord injured because the existence of the pressure ulcer increases the length of stay and degrades the quality of life. Its incidence in acute phase is high $40 \% .^{3}$ The very early mobilization of paraplegics and quadriplegics most probably contributes to reducing the complications of decubitus and especially pressure sores. $94 \%$ of spinal cord injuries were treated at least once for a low urinary tract infection while $3.5 \%$ had pyelonephritis. The rate of orchiepididymitis is evaluated between 5 and $28 \%$ in longterm follow-up cohort studies (over one year). ${ }^{4,5}$ The incidence of prostitutes was estimated to be between 5 and $18 \%$ of the spinal cord injury population. ${ }^{5}$ In the quadriplegic patient, the first reason for readmission was urinary infection ( $43 \%$ for Vaidyanathan ${ }^{6}$ and $32 \%$ for $\mathrm{Klotz}^{7}$ ). Infectious risks, both urinary and systemic, appear to be more important in quadriplegics and complete paraplegics. ${ }^{8}$ The usual behavior is not to treat asymptomatic bacteriuria although they are associated with an increased risk of symptomatic urinary tract infections. ${ }^{9}$
Early treatment of spasticity can be justified if spasticity is severe and limits joint mobilization or patient installation. The place of oral treatment is low in the early phase The efficacy of baclofen, tizanidine and dantrolene is mediocre and counterbalanced by side effects. Baclofen, a GABAergic agonist, may have a deleterious effect on neurological recovery. The therapeutic arsenal of the early phase is currently based on a focal or regional treatment of spasticity with botulinum toxin; Chemical neurolyses with alcohol or nerve trunk phenol purely motors are less and less practiced. ${ }^{10}$ Baclofen injected intrathecally is an effective treatment of diffuse spasticity of the lower limbs. Its use is common in rehabilitation: after one or more injections tests carried out by the lumbar way, a pump allowing a continuous diffusion of the product is implanted. ${ }^{11}$ Neurogenic paraosteoarthropathy is most common in spinal cord injuries (13 to $20 \%$ of subjects ${ }^{12}$ ). They preferentially affect the large joints, especially the hips. Our study showed only $8 \%$ of paraosteoarthopathy with predominance in the hip joint.

The fight against joint stiffness is essential as ankylosis can then compromise the functional prognosis of the patient. The correct installation of the subject in order to limit the stiffness in the vicious position (particularly the equine foot) must be constant. Multipleday mobilizations are clinically evident in terms of effectiveness in combating joint stiffness but there are little published on this subject. ${ }^{13}$ In case of stiffness, the posture allows to improve the passive articular amplitudes. The specific management of the spasticity also makes it possible to limit the stiffness's. The robotic mobilizations of the paretic members are still mainly reserved for later phases of recovery. ${ }^{14}$

In the spinal cord injury pain is common with an average prevalence of $69 \%{ }^{15}$ and have a major impact on the everyday life constituting a major obstacle to autonomy quality of life and integration into the community. The treatment of pain, despite the difficulties of its evaluation, follows the traditional recommendations: analgesics will be chosen in the different levels according to the intensity of the pain, and in case of neuropathic pain, tricyclic antiepileptics or antidepressants can be introduced. ${ }^{14}$ In our study, neuropathic pain occurred in $4 \%$ of patients.

Dysautonomia is particularly common after high spinal trauma: in response to a stimulus, usually painful, there is hyperactivation of the sympathetic system. Clinically, there is an increase in blood pressure, heart rate and respiratory rate, hypersudation. The treatment is based essentially on the suppression of the cause with symptomatic treatment. ${ }^{12}$

The incidence of thromboembolic complications is high in spinal cord injuries. Several additional risk factors were identified: age over 40 years, immobility and/or paralysis, presence of a central venous catheter, orthopedic trauma to the lower limbs and history of thromboembolic disease. ${ }^{16}$ Prevention by low-molecular-weight preventive heparin is necessary in the absence of contraindication. For elastic restraints of the lower limbs, evidence of their efficacy was based on randomized trials of small series, and on patients in postoperative orthopedic surgery. ${ }^{14}$ This efficacy has recently been called into question by a study which shows that the use of compression stockings, on the other hand, increases the skin risk. ${ }^{14}$

\section{Conclusion}

Spinal trauma is a frequent pathology of the young adult, which compromises the vital and functional prognosis. The objective of the management of spinal cord injuries and their complications is to 
improve the provision of care proposed and especially to articulate it with the expectations of the patient confronted with his new life. Our study is the first to determine the sociodemographic and clinical characteristics of patients with spinal cord injury in Morocco. It has some limitations as it was a retrospective study, it was monocentric and the sample was not very large and was constituted principally by a military population. Further studies, prospective and multicentre studies with a larger sample are needed.

\section{Acknowledgements}

None.

\section{Conflict of interest}

There is no conflict of interest among the authors.

\section{References}

1. Bauchet L, Lonjon N, Fattal C. Novelties in traumatic bone marrow lesions. France: Hellemmes center; 2011.

2. Fattal C, Rouays MH, Verollet C. Rehabilitation of acquired spinal cord injury in adults: ASIA A. EMC. Physiotherapy Physical Medicine Rehabilitation. 2010;26(460A):60.

3. Gélis A, Dupeyron A, Legros P, et al. Of ulcerative colitis in patients with SCI: part I: acute and rehabilitation stages. Spinal Cord. 2009;47(9):99107.

4. KJ Weld, Dmochowski RR. Effect of bladder management on urological complications in spinal cord injuried patients. $J$ Urol. 2010;163(3):768772

5. JJ Wyndaele. Complications of intermittent catheterization: their prevention and treatment. Spinal Cord. 2000;40(10):536-541.
6. S Vaidyanathan, BM Soni, L Gopalan, et al. A review of readmissions of patients with tetraplegia to the regional injuries center, southport, United Kingdom, between January 1994 and December 1995. Spinal Cord. 1938;36:838-846.

7. R Klotz, PA Joseph, JF Ravaud, et al. The Tetrafigap. The complications and associated factors. Spinal Cord. 2002;40(9):457-467.

8. PA Joseph, M de Seze, L Soyeur. Treatment of nosocomial urinary tract infections in patients with a neurogenic bladder. Medicine and Infectious Diseases. 2003;33:488-497.

9. H Eden, S HIzmetli, V Nacitarhan, et al. Relapsing bacteriuria in patients with spinal cord injury. Arch Phys Med Rehabil. 1997;78(5):468-470.

10. A fssaps. Medicinal treatments of spasticity. good practice; 2009.

11. D Guillaume, A Van Havenbergh, M Vloeberghs, et al. A clinical study of intrathecal baclofen using a programmable pump for intractable spasticity. Arch phys Med Rehabil. 2005;86(11):2165-2171.

12. Hendricks HT, Heeren AH, Vos PE. Your dysautonomia after severe traumatic brain injury. Eur J Neurol. 2010;17(9):1172-1177.

13. Hellweg S, Johannes S. Physiotherapy after traumatic brain injury: a systematic review of the literature. Brain Inj. 2008;22(5):365-373.

14. L Oujamaa, A Marquer, G Francony, et al. Early rehabilitation for neurologic patients. Ann Anais of the Anesth Réa. 2012;31:253-263.

15. PJ Sidall, RP Yesierski, JD Loeser. Bone marrow transplantation: clinical features, prevalence and taxonomy. IASP news letter. 2000;(3).

16. KS Kim, GM Brophy. Symptomatic venous thromboembolism: incidence and risk factors in patients with spontaneous or traumatic intracranial hemorrhage. Neurological Care. 2009;11(1):28-33. 